Miley, G.H. and P. Shrestha. Review Of Transmutation Reactions In Solids. in Tenth International Conference on Cold Fusion. 2003. Cambridge, MA: LENR-CANR.org. This paper was presented at the 10th International Conference on Cold Fusion. It may be different from the version published by World Scientific, Inc (2003) in the official Proceedings of the conference.

\title{
Review Of Transmutation Reactions In Solids
}

\author{
G. H. MILEY AND P. J. SHRESTHA \\ Department of Nuclear, Plasma, and Radiological Engineering, University of Illinois, \\ Urbana-Champaign Campus, 103 S. Goodwin, Urbana, IL 61801 USA \\ E-mail: g-miley@uiuc.edu
}

\begin{abstract}
Transmutation reactions in highly loaded hydrides have been reported by a number of research groups. These studies are briefly summarized with emphasis on common systematics and key "signatures". Transmutations divide into two types: heavy intermediate compound nucleus reactions yielding an array of products with a large spectrum of masses; direct reactions between $\mathrm{H} / \mathrm{D}$ and the electrode metal or impurity atoms yielding isolated "single" products. Various mechanisms have been proposed to explain the products and the ability to overcome the extremely large Columbic repulsion of the high- $Z$ elements involved. Here we briefly consider a model involving orbital mixing and virtual neutron formation associated with charge accumulation and hydrogen/deuteron flow at highly loaded interfaces.
\end{abstract}

\section{Introduction}

A variety of Low Energy Nuclear Reactions (LENRs) in solids have been reported. The original PonsFleishmann (P-F) "cold fusion" reaction involved D-D fusion, but instead of following the normal D-D reaction channels, the intermediate product $\mathrm{He}^{4}$ was de-excited by energy transfer to the lattice that results in heat (Table 1). Also "transmutation" reactions have been reported that involve an interaction between the D (or H) with atoms in the host lattice, typically heavy metals. In some P-F type experiments the tritium branch rates have been enhanced [1], but here we concentrate on reactions involving host metal atoms.

Table 1. LENRs differ from "normal” D-D fusion and can be divided into P-F type D-D and D/H-metal reactions.

\begin{tabular}{|c|c|c|c|}
\hline \multirow{4}{*}{$\frac{\text { D-D Reaction }}{\text { D-D }}$} & Reaction Products & \multicolumn{2}{|l|}{ Comments } \\
\hline & $\mathbf{T}+\mathbf{p}$ & \multirow{2}{*}{\multicolumn{2}{|c|}{$\begin{array}{l}\text { Each branch } 50 \% \text { probable } \\
\text { "Normal" D-D Fusion }\end{array}$}} \\
\hline & $\mathrm{He}-3+\mathrm{n}$ & & \\
\hline & He-4 + hv (heat) & "P-F type Cold Fusion" & \multirow[b]{2}{*}{ LENR } \\
\hline$\frac{\text { Lattice Reaction }}{\text { p/D + metal }}$ & $\begin{array}{c}\text { Isolated Isotopes or "fission" product } \\
\text { array }\end{array}$ & "Transmutations" & \\
\hline
\end{tabular}

\section{Types of Transmutation Reactions}

Transmutation reactions can be broadly classified according to their characteristic products. Some experiments give a large array of reaction products with mass numbers spanning across the periodic table. These reactions are traced to multi-body events leading to formation of a heavy compound nucleus, which can both decay and fission into an array of elements [e.g. see Ref 2]. The second reaction class leads to distinct isolated products [e.g. see Ref 3 and 4]. These reactions may or may not involve multi-body interactions but the net result is direct formation of the products vs. the disintegration of a compound nucleus. We will first briefly review reactions leading to an array of products and then consider "single" product reactions.

\subsection{Transmutation experiments giving a broad array of products.}

An array of reaction products were reported as early as 1992 by Karabut et al. [5] in experiments using a glow discharge to bombard and load various targets such as Pd. Later experiments leading to product arrays include those by Bockris and Minevski [6], Mizuno et al. [7-9], and Miley et al. [2]. These experiments used electrolytic methods to load a cathode but others have used different techniques, e.g. a UV laser irradiation of deuterated films by Castellano et al. [10]. Here we briefly review the Miley-Patterson work, which is 
illustrative of this class of transmutation reactions. They employed multi-layer thin-film Ni/Pd coated on mm diameter plastic spheres loaded to a high ratio of $\mathrm{H} /$ film-metal atoms using a packed-bed-type electrolytic technique. One distinguishing feature of this work was the use of combined SIMS-NAA diagnostics to obtain quantitative measurements of selected product yields. The NAA analysis probed the total sample volume and was calibrated against the NIST standards.

A distinctive characteristic of these product arrays is a yield vs. mass curve with four regions of high yield peaks (A 22-23, 50-80, 103-120 and 200-210). This pattern is generally consistent with results by Bockris and Minevski [6] and Mizuno et al. [7-9]. This product array is illustrated later in Figure 4. Higher yield elements occurred in quantities well above maximum impurity limits, which include possible accumulation of isotopes on the cathode due to electrolytic field effects. Consistent with a nuclear reaction source, non-natural isotopic distributions were found for select elements. In addition, recent CR-39 track detector measurements have revealed low-level emission of $\sim 1.6 \mathrm{MeV}$ protons and $\sim 16 \mathrm{MeV}$ alpha particles from similar thin-film electrodes [11-12]. Attempts to explain these observations theoretically have expanded the original swimming electron layer (SEL) theory that leads to the use of the multi-layer thin-film electrodes [13].

Recent studies at the UIUC have converted from microspheres to thin films sputtered onto a flat alumina substrate illustrated conceptually in Figure 1. In this design the electrical current flow is parallel to the thin-film interface to provide a high current density and hydrogen flow rate along with a high loading. Results from this work, including calorimetry and charged particle emission measurements, were summarized in [14].

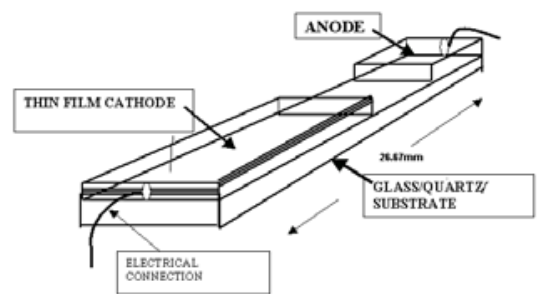

Figure 1. Recent UIUC work uses a unique integrated thin-film plate-type electrode.

\subsection{Single product "direct" type transmutation.}

Early electrolysis based transmutation experiments frequently reported one or more isolated products, i.e. "direct transmutations" vs. product arrays [e.g. see Ref 15, 16 and 17]. In several cases radioactive products were observed [15]. Most recently Mitsubishi Corp. researchers reported a precision real-time measurement using built-in XPS diagnostics. An atomic layer of Sr-88 coated on a thin-film Pd substrate was transmuted into Mo-96 over 200 hours (Figure 2). The experiment used 1 atm of deuterium on one side of the thin film to force deuterium diffusion through a multi-layer thin-film $\mathrm{Pd} / \mathrm{CaO}$ substrate [3]. Cs-133 was also transmuted into $\mathrm{Pr}$ 141 in a similar experiment. 


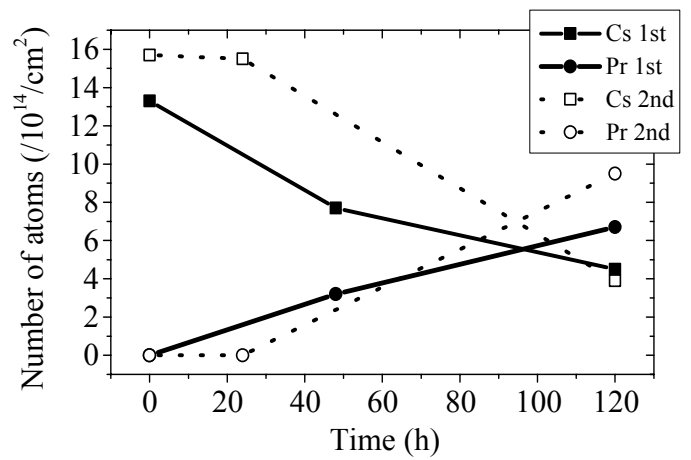

(a)

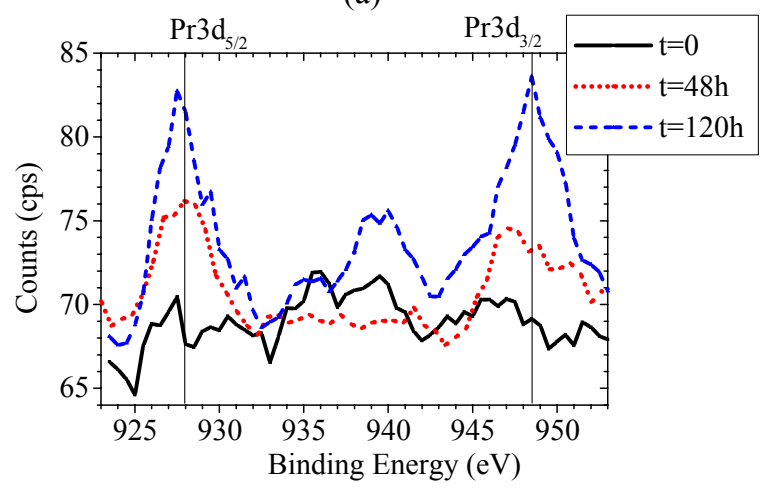

(b)

Figure 2. Experimental results obtained by $\mathrm{D}_{2}$ gas permeation through $\mathrm{Pd}$ complex ( $\left.\mathrm{Pd} / \mathrm{CaO} / \mathrm{Pd}\right)$ deposited with Cs: (a) Time variation in number of Cs and Pr atoms, (b) XPS spectrum of Pr for experiment run \#1.

Preferential build up of Mo-96 was observed, representing a significant deviation from natural isotopic abundance of Mo. ( $\mathrm{Pr}$ only has one isotope). In both cases the mass and charge changes by 8 and 4 , respectively. In other words, the "single" product reactions involved are:

A confirmation of this type of experiment was recently reported by Yamada et al. [18], but instead of an

$$
4_{1}^{2} \mathrm{D}+{ }_{55}^{133} \mathrm{Cs} \rightarrow{ }_{59}^{141} \mathrm{Pr} \quad 4_{1}^{2} \mathrm{D}+{ }_{38}^{88} \mathrm{Sr} \rightarrow{ }_{42}^{96} \mathrm{Mo}
$$

atomic coating on the film, they studied other transmutations in a thicker Pd foil using both $\mathrm{H}$ and $\mathrm{D}$ diffusion.

\section{Overview of Worldwide Studies}

Transmutation experiments have been studied in some detail by over 14 separate laboratories worldwide. This listing is incomplete but hopefully representative. It includes Beijing University, China [19], Tsinghua University, China [20], Lab des Sciences Nucleaires, France [21], Frascati Laboratory, Italy [22, 23], University of Leece, Italy [24], Hokkaido University, Japan [25, 7], Mitsubishi Corporation, Japan [4], Osaka university, Japan [26, 27], Shizuoka University, Japan [28], SIA LUTCH, Russia [29, 30], Tomsk Polytechnical University, Russia [31], Portland State University, USA [32], Texas A \& M University, USA [6] and UIUC, USA [33]. (Most of the references cited represent a recent publication from the lab). Other reviews of transmutation studies by Dash [34] and Kozima [28] also confirms the many experimental results reported worldwide. The fact that this number of major laboratories report positive results provides further confidence that LENR-type transmutation reactions can occur with some predictability in solids.

Experiments have used a wide variety of conditions, e.g. different electrode materials and loading methods. Electrode materials have ranged from carbon [27], nickel [35] to uranium [21]. Various loading methods have been used: electrolysis [e.g. see Ref 2], surface plasma electrolysis [e.g. see Ref 7], gas pressure [e.g. see Ref 3], plasma discharge [e.g. see Ref 29], ion implantation [e.g. see ref 36], bubble collapse loading [e.g. see ref 37] 
and laser initiation [e.g. see Ref 24]. Thus it appears that the basic physics underlying these reactions involves obtaining adequate loading and flow of $\mathrm{D}($ or $\mathrm{H})$ in a metallic lattice that can undergo the desired transmutation. This can be done using a wide variety of loading methods and electrode designs, as shown by the wide range of successful experimental cited here.

Some indication of the variety of products involved is illustrated in Figure 3, which summarizes the frequency of observation reported for various elements, including both array and direct type reactions.

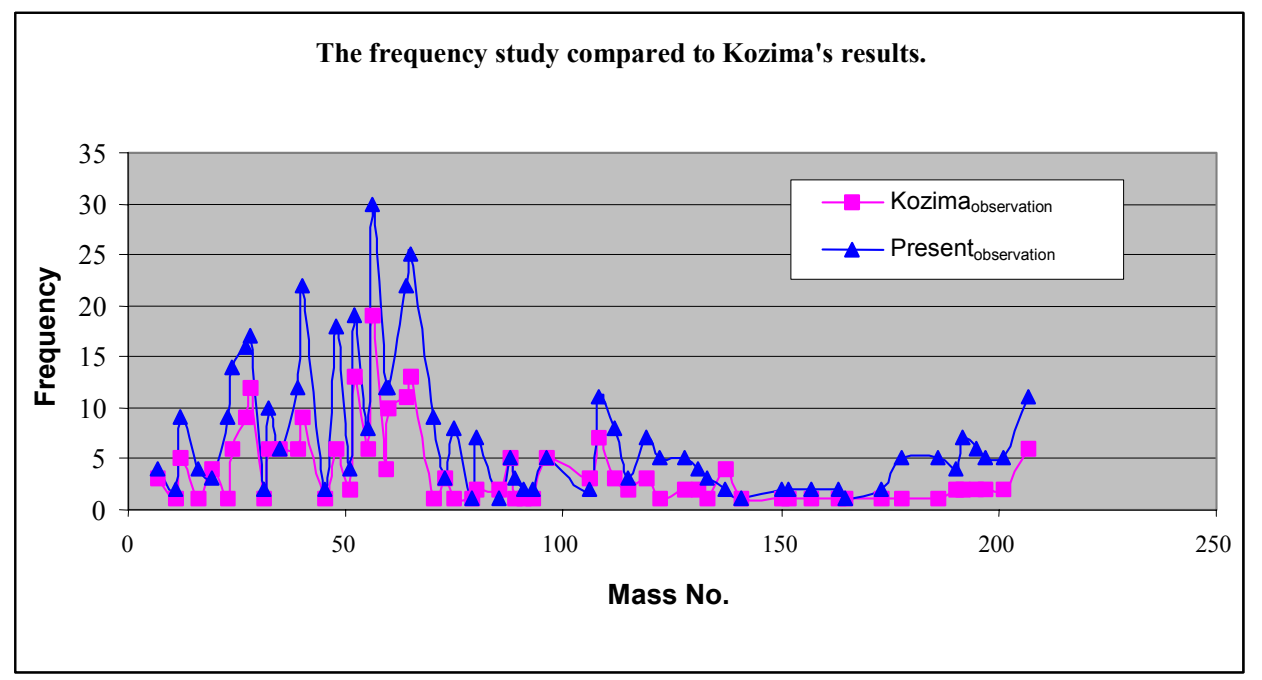

Figure 3. Frequency of observation of elements vs mass number for 48 references in the present study (see Appendix A for references citation). Also shown are frequencies reported by Kozima [28] from 40 references.

The frequency of observation found here is consistent with that reported independently by Kozima [28], also shown in Figure 3. Good agreement between the results from these two independent studies is observed. The most commonly reported elements are Calcium, Copper, Zinc and Iron. They were observed in more than 20 different experiments. $40 \%$ of the least frequently observed elements were rare earth elements from the Lanthanide Group. These included: Lutetium, Terbium, Praseodymium, Europium, Samarium, Gadolinium, Dysprosium, Holmium, Neodymium and Ytterbium. The observation of these rare earth elements is particularly significant since they are unlikely to enter as impurities. Some researchers also performed detailed analyses to determine possible isotopic deviations from the natural abundance. Results from more detailed measurements of this type are tabulated in Table 2 . 
Table 2. Percentage deviation from natural isotopic abundance for select reaction products.

\begin{tabular}{|c|c|c|c|c|c|c|c|}
\hline Isotopes & $\begin{array}{c}\text { Mizuno et al. } \\
{[8]}\end{array}$ & ${ }_{\text {[35] }}^{\text {Miley et al. }}{ }^{a}$ & $\begin{array}{c}\text { Ohmori } \\
\text { et al. } \\
\text { [38] }\end{array}$ & $\begin{array}{c}\text { Ohmori } \\
\& \\
\text { Mizuno } \\
{[39]}\end{array}$ & $\begin{array}{c}\text { Chernov } \\
\text { et al. } \\
{[31]}\end{array}$ & $\begin{array}{c}\text { Ohmori } \\
\& \\
\text { Mizuno } \\
{[40]}\end{array}$ & $\begin{array}{c}\text { Ohmori } \\
\text { et al. [41] }\end{array}$ \\
\hline & 1996 & 1997 & 1997 & 1998 & 1998 & 2000 & 2002 \\
\hline$K-39$ & & & & & & & $-21.33 *$ \\
\hline$K-41$ & & & & & & & 21.33* \\
\hline Cu-63 & 30 & $3.6 \pm 1.6$ & & & 44 & & \\
\hline Cu-65 & -30 & $-8.1 \pm 3.6$ & & & -44 & & \\
\hline $\mathrm{Fe}-54$ & $* *$ & & $-0.81 *$ & -0.8 & & & \\
\hline $\mathrm{Fe}-56$ & -21.75 & & $-16.79 *$ & -29.72 & & & \\
\hline $\mathrm{Fe}-57$ & 18.88 & & $16.58 *$ & 30.8 & & & \\
\hline $\mathrm{Fe}-58$ & $* *$ & & $1.01 *$ & $* *$ & & & \\
\hline$R e-185$ & 14.60 & & & & & 11.3 & \\
\hline $\operatorname{Re}-187$ & -14.60 & & & & & -11.3 & \\
\hline Ag-107 & & $3.9 \pm 1.2$ & & & & & \\
\hline Ag-109 & & $-4.3 \pm 1.3$ & & & & & \\
\hline$T i-48$ & & & & & -33.30 & & \\
\hline$T i-49$ & & & & & 8.60 & & \\
\hline$T i-50$ & & & & & 17.80 & & \\
\hline$C r-50$ & & & & & & 1.79 & \\
\hline Cr-52 & & & & & & -4.65 & \\
\hline Cr-53 & & & & & & 2.85 & \\
\hline$N i-58$ & & & & & & -4.98 & \\
\hline $\mathrm{Ni}-60$ & & & & & & 4.57 & \\
\hline$P b-206$ & & & & & & 13.5 & \\
\hline$P b-207$ & & & & & & 33 & \\
\hline$P b-208$ & & & & & & -6.5 & \\
\hline
\end{tabular}

* Averaged value from the reference paper; ** data not provided. ${ }^{a}$ Performed by NAA such that error bars account for discrepancy in \pm numbers (equal for two isotopes, sum to zero for multiple isotopes).

Due to the large differences in conditions and uncertainties involved in these experiments, quantitative agreement might not be expected. However the fact that the trends (i.e. isotopic percentages increasing/decreasing) are similar for elements where overlapping data exists $(\mathrm{Cu}, \mathrm{Fe}$, and $\mathrm{Re})$ are consistent with a common reaction mechanism, although the data is too sparse to allow firm conclusions.

There were other associated effects observed along with transmutation products. These include energetic charged-particle, protons and alphas emission [11-12, 36, 42-44] and low level soft X-ray emission [11,12,23]. While there appears to be a direct relation between the transmutation reactions and the energetic charged particles, the reports by Fisher $[43,44]$ that particles originate in the electrolyte raise questions about the relationship. Excess heat production was also observed simultaneously with transmutations in various experiments [2,7-9]. Thus the production of excess heat using D/H-metal reactions appears feasible with proper selection of conditions and materials. Miley [45] has studied the relationship between excess heat production and the observed product array in select experiments. Based on a basic binding energy calculation he concludes that the product production rate correlates with the excess power measured within the experimental accuracy.

Dash et al. [46], Mizuno [47] and others have reported finding transmutation products in small regions around "volcanic-like" surface structures. Others e.g. Miley et al [35] have not observed this phenomenon although such structures have been found on some of their electrode structures [48]. How, or if this phenomena is connected to the reaction process, is unclear. Shoulders [49], Fox and Baraboshkin [50] associate it with possible $\mathrm{eV}$ (electron cluster) formation which they view as driving the reaction. In contrast Miley views the volcanoes as reactive regions where reaction conditions are highly favorable (possibly related to the "nuclear reactive regions" postulated by Storm [51], causing extremely violent reactions rates that cause local melting before being extinguished. If so, the thin-film surface conditions created in the Miley et al. experiments appear 
to create a reasonably uniform active region over the surface. This is partially confirmed by SIMS surface scans of key products such as $\mathrm{Cu}$, which were generally uniform within $\pm 10 \%$ over the surface [35].

Surface conditions and the active region thickness clearly play an important role in reactive regions. For example Miley et al. report that isotope concentrations typically peak near multi-layer interfaces [35]. The electrolyte also plays an important role. Transmutations have been obtained with both light and heavy water based solutions. The optimum combination appears to depend on the selection of an appropriate electrodeelectrolyte combination. For example, Castellano et al. [10] observed an increase in the number of transmutation products, as a function of the thickness of a solid metal electrode Di Guilio et al. [52] deduced that compared to light water electrolysis, heavy water solutions give a larger number of transmutation products for their configuration.

\subsection{Reaction Systematics}

Until a theoretical model is completely worked out, only some generalities can be said about reaction systematics. "Direct" reaction systematics are more easily envisioned than for compound nucleus reactions. Once the light element ( $\mathrm{D}$ or $\mathrm{H}$ ) multiple involved is determined, the reaction immediately follows, e.g. as reported by Bush and Eagleton for a single $\mathrm{H}$ reaction [16].

or multiple (four) D reactions provided the Pr or Mo products reported by Iwamura et al. [3], as cited earlier.

$$
{ }_{1}^{1} \mathrm{H}+{ }_{37}^{85} \mathrm{Rb}\left({ }_{37}^{87} \mathrm{Rb}\right) \rightarrow{ }_{38}^{86} \mathrm{Sr}\left({ }_{38}^{88} \mathrm{Sr}\right)
$$

In contrast in the case of the compound nucleus reaction, there is no simple prediction of either the product array or degree of deviation from natural isotopic abundance. Hora et al. [53] have discussed some guiding principles related to magic numbers and point out some similarities to the r-process occurring in astrophysical reactions. This is illustrated in Figure 4 where the Miley-Patterson product array [2] is compared to r-process calculations for astrophysical reactions [54]. As pointed out by Hora et al. [53,55-58], the magic number sequence and high density lattice conditions suggest a relationship between the reactions involved in compound nucleus formation and the r-process. While there are some differences, the similarity in trends is striking.

Other predictions of product distributions have generally involved adaptation of a fission model (e.g. liquid drop model) to the compound nucleus [26, 59]. However, these theories differ greatly in the mechanisms assumed in the formation of the compound nucleus, hence the characteristic $\mathrm{A}, \mathrm{Z}$ and excitation energy of the intermediate state leads to the products.

Kozima [28] has pointed out the similarities between the frequency of observation of elements and element abundance in nature. Thus he concludes LENR reactions for a path similar to the creation of natural elements. However this raises the issue of the relationship between observation frequency over disparte experiments and actual yields in a given experiment. As it turns out, the composite observation frequency roughly follows the trend of the experiment yield curve in Figure 4. Hence Kozima's comparison ends up roughly resembling Figure 4, but the basis remains quite different. 


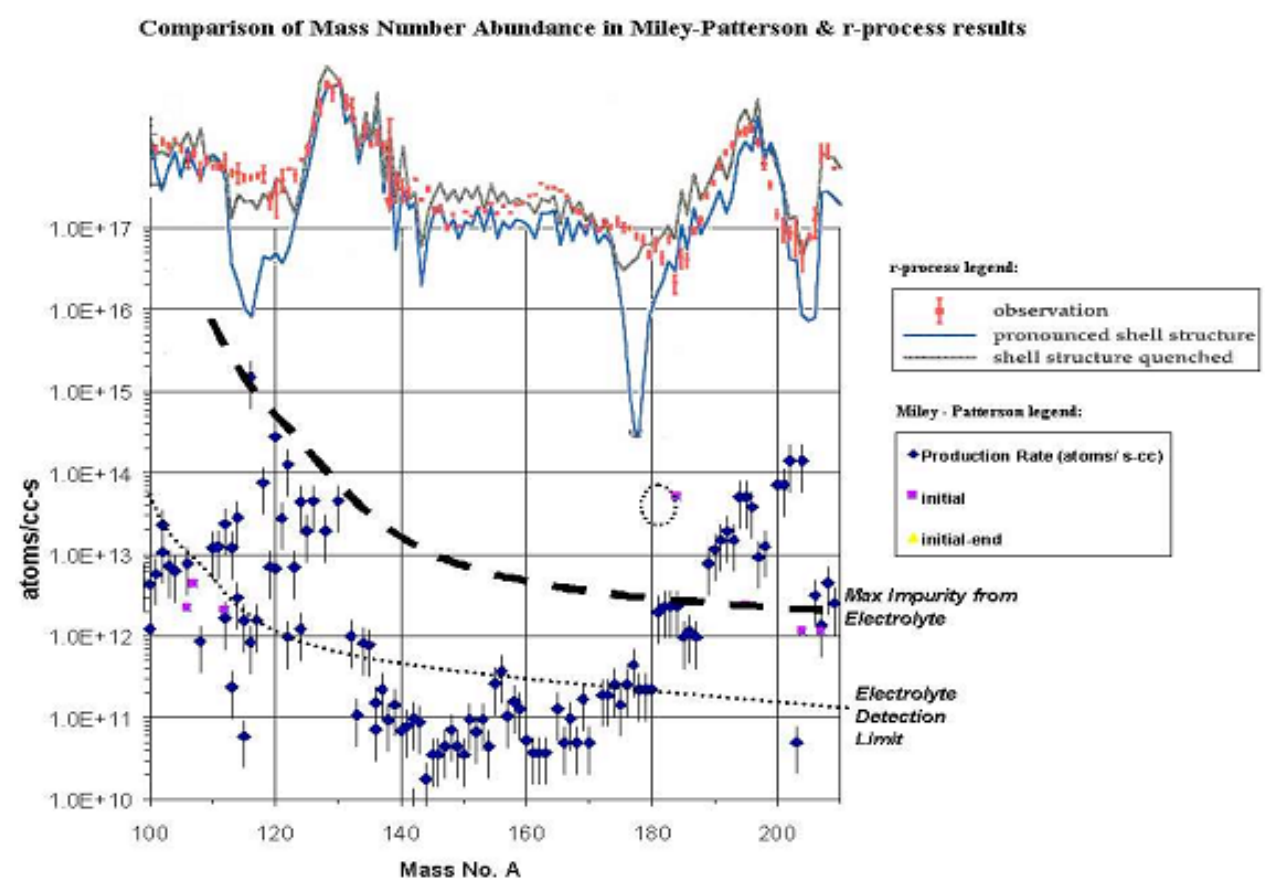

Figure 4. Comparison of yield vs. mass number in Miley-Patterson [2] to r-process predictions.

\section{Theory}

An obvious key issue for transmutation theories is how the reactants can overcome the extremely large Coulombic barrier. The same issue applies to P-F type D-D reactions, but here the atomic number of the metal atom involved is over an order of magnitude greater. Thus the theories have tended to focus on neutral-like particles (e.g. poly- neutrons of Fisher [59], Kozima [28], hydrex catalyzed rx of Dufour et al. [60], electron capture of Stoppini [61], lattice condensation of Takahashi et al. [26]).

One approach recently studied by G. Miley et al. extends SEL concepts to include orbital mixing associated with charge accumulation and hydrogen/deuteron flow at highly loaded interfaces $[62,63]$. These phenomena result in formation of virtual neutrons, overcoming the columbic repulsion barrier restriction. The flow path for the resulting reaction is very complicated, leading through a compound nucleus and subsequent decay or fission.

Negative $\mathrm{H}$ formation is thought to play an initiating role in this process [14,62,63-65]. The issue of the charge state of $\mathrm{H}$ diffusing in $\mathrm{Pd}$ was originally motivated by our attempt to reconcile the static picture of a negative charge accumulation around $\mathrm{H}$ in Pd with the fractionally charged positive $\mathrm{H}$ ion found in drift experiments [65]. The effect is now attributed to the dynamic nature of the hydrogen hopping process. When an electric field is applied to the $\mathrm{H}^{\delta-}$ at the octahedral site ( $\delta$ - is the fractional electronic charge), the hydrogen hopping (tunneling) process is driven in the direction of the field. Therefore, the hydrogen drift (or in other words, an electric field assisted tunneling process) is characterized as that of a proton, i.e., a +1 charge, at the octahedral site. However, in hopping from one octahedral site to another, the hydrogen must follow a path through either the tetrahedral site or the bridging site, and the charge state at these two locations will modify the time average charge measured in a drift experiment. The path through the bridge site is the shortest of the two available. The charge density as calculated from a density functional method is summarized in Table 3 . The $\mathrm{H}$ is roughly charged with 0.6 more electrons at the bridging site than at the octahedral site. This implies that the $\mathrm{H}$ carries an effective (time-averaged) electron cloud of -0.6 as it "hops" through the bridging region. Hence the net charge manifested in this process is $(1-0.6)$ or $\sim 0.4$, roughly matching the typical value of 0.5 found in drift measurements. 
Table 3. The charge in the Muffin-Tin Sphere around the $\mathrm{H}$ is much larger for edge (bridging) site than for other sites (corresponding to fractional loadings). The radius of the muffin-tin sphere is taken as $1.5 \mathrm{Bohr}$.

\begin{tabular}{|c|c|c|c|c|}
\hline Case & $\mathrm{PdH}$ & $\mathrm{PdH}_{0.25}$ & $\mathrm{PdH}_{0.5}$ & PdH (Edge) \\
\hline Charge & 1.22 & 1.13 & 1.18 & 1.81 \\
\hline
\end{tabular}

Due to the high local charge density that forms during this hopping process, orbital mixing can occur, facilitating virtual neutron formation. Virtual neutrons in turn enable a reaction with one or more neighboring Pd-H sites. At high density, a cluster of neighboring sites can undergo formation of a large mass compound nucleus. The concept has also been extended to the case of an atomic layer of Cs on a Pd surface undergoing D diffusion as in the Iwamura et al. experiment [3] based on the lattice configuration shown in Figure 5. The hypothesis is that during flow through the constricted path between the $\mathrm{Pd}$ and $\mathrm{Cs}$, the highly negatively charged H (D) at the four "edge sites" condensates into a virtual neutron and then reacts with the large Cs. A "direct" reaction occurs in this case due to the combination of the edge site location and the size and electronegatively of the Cs which partly fills the hoping site passages. In this case, flow is more important than a high D/H loading. The Cs blocking plays the role of a high loading. In contrast, if, in a more open $\mathrm{Pd}$ (or $\mathrm{Ni}$, etc.) lattice (Figure 5b), the loading is sufficiently high, the diffusing H (D) causes passage blocking (a "selfblocking") and allows entire clusters of virtual neutron Pd cells to condense into a heavy compound nucleus. This heavy nucleus can either decay directly or fission into a broad array of reaction products.

In summary, this model predicts direct reactions in cases where appropriate heavy elements tend to compress flow channels during $\mathrm{H}$ (D) diffusion while heavy compound nucleus formation occurs where the $\mathrm{H}$ (D) loading is so large as to compress flow during diffusion.
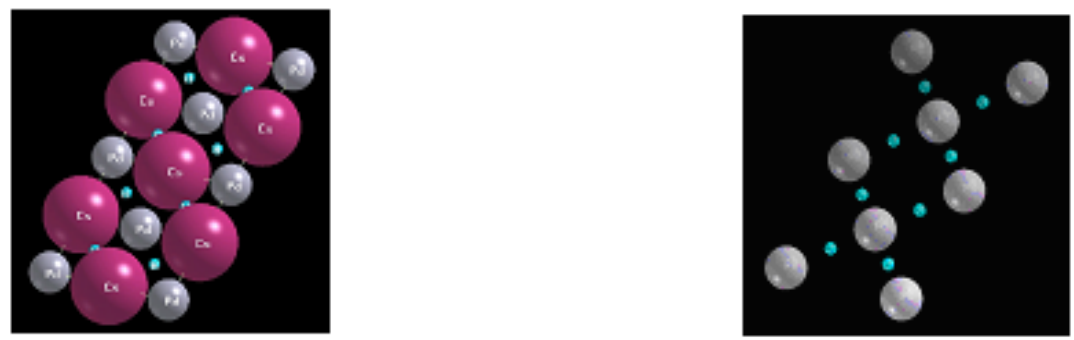

(b)

Figure 5. Figure (a) shows two surface unit cells to represent the case of an atomic layer of Cs on the Pd surface. It is seen that each Cs is surrounded by $4 \mathrm{H}$ (D). Figure (b) contrasts the more open lattice formed by H/D loading in Pd.

\section{Summary}

Several dozen laboratories worldwide are actively pursuing the study of transmutations in low energy nuclear reactions. Their methodology and choice of electrode material are quite varied but still their results demonstrate many related trends. Products are either found to occur in arrays covering a wide range of masses or in a few isolated elements, depending on experimental conditions. The most commonly reported elements are Calcium, Copper, Zinc and Iron, while $40 \%$ of the least frequently observed elements are from the Lanthanide group. Some studies also identify products with a significant deviation from the natural abundance.

Various associated effects such as radiation emission have been observed in some experiments, but a direct relationship to specific nuclear reactions causing transmutation has yet to be established. Also, in some experiments excess heat production has been reported, confirming that in these cases the overall transmutation reaction is exothermic. Correlation of the reaction energy (i.e. the transmutation product array reaction rates) with calorimetric measurements of excess heat is difficult. However, several cases indicate a correlation within experimental error bars [45]. This result is analogous to the correlation of He-4 production and excess heat production in D-D cold fusion studies [66].

Various theories have been proposed to explain how the high- $Z$ reactants in transmutations can overcome the gigantic Coulombic barrier involved and why isolated product reactions occur in some cases and arrays of 
products in others. A preliminary model that considers orbital mixing associated with charge accumulation and hydrogen/deuteron flow at highly loaded interfaces was reviewed as providing one possible explanation. The subsequent formation of a virtual neutron, overcomes the Columbic repulsion barrier restriction. Depending on the lattice structure and the species that restricts the hopping passages during diffusion of the $\mathrm{H}$ (D), either an array of products or single element products is predicted. Other theories cited were also address these issues but in quite different ways. As more data and study is preformed, it should be possible to sort out a theory or combination of theories that best reproduce experimental signatures.

In conclusion, numerous observations of transmutation reactions have been reported by a variety of laboratories internationally, lending much strength to the conclusion that transmutation reactions can take place in solids under select conditions. A key question remains on how it is possible to design a specific experiment that would emphasize one of these reaction product channels over the others. Steps along these lines include the technique demonstrated by Iwamura et al. [3] to use atomic surface layers on top of a thin-film complex and the technique of Miley et al [14] of using selective multiple layers of thin-films with directed current flow along the interface direction.

\section{Acknowledgments}

The willingness of many researchers cited here to provide data is gratefully acknowledged. Contributions by colleagues at the UIUC, A. Lipson and N. Luo, are also greatly appreciated. Some of the UIUC work reported was supported by Lattice Energy LLC.

\section{References}

1. T. N. Claytor, M.J. Schwab and D.J. Tuggle, "In-situ Measurement of Tritium concentration Variations during Plasma Excitation of Deuterium Loaded Palladium and Palladium Alloys," ICCF7-Abstracts, Vancouver, Canada, p. 48, Apr 19-24 (1998).

2. G.H. Miley, and J.A. Patterson, "Nuclear Transmutation in Thin-film Coatings Undergoing Electrolysis," $J$. New Energy, vol. 1, pp.11-15, (1996).

3. Y. Iwamura, M. Sakano, and T. Itoh, "Elemental Analysis of Pd Complexes: Effects of D2 Gas Permeation," Japan Soc. App. Phys., vol. 41, Pt. 1, no. 7A, pp.4642-4650, (2002).

4. Y. Iwamura, T. Itoh, M. Sakano, S. Sakai and S. Kuribayashi, "Low Energy Transmutation in Condensed Matter induced by D2 Gas Permeation through Pd Complexes: Correlation between Deuterium Flux and Nuclear Products," ICCF-10 Abstracts, Cambridge, MA, p. 68, Aug 24-29 (2003).

5. A.B. Karabut, Y.R. Kucherov and I.B. Savvatimova, "Possible Nuclear Reactions Mechanisms at Glow Discharge in Deuterium," ICCF-3, Nagoya, Japan, p. 165, Oct 21-25 (1992).

6. J. O'M. Bockris, and Z. Minevski, "Two Zones of "Impurities" Observed after Prolonged Electrolysis of Deuterium on Palladium," Infinite Energy, no. 5 \& 6, pp. 67-73, Nov (1995).

7. T. Mizuno, T. Ohmori, T. Akimoto, K. Kurokawa, M. Kitaichi, K. Inoda, K. Azumi, S. Simokawa and M. Enyo, "Isotopic Distribution For The Elements Evolved In Palladium Cathode After Electrolysis in $\mathrm{D}_{2} \mathrm{O}$ Solution," ICCF-6, Hokkaido, Japan, pp.71, Oct 12-13 (1996).

8. T. Mizuno, T. Ohmori and M. Enyo, "Anomalous Isotopic Distribution in Palladium Cathode after Electrolysis," Infinite Energy, vol.2, no.7, pp.10-13, (1996).

9. T. Mizuno, T. Ohmori and M. Enyo, "Isotopic Changes of the Reaction Products Induced by Cathodic Electrolysis in Pd," J. New Energy, vol. 1, no. 3, pp. 31-45, (1996).

10. Castellano, M. Di Giulio, M. Dinescu, V. Nassisi, A. Conte, and P.P. Pompa, "Nuclear Transmutation in Deuterated Pd Films Irradiated by an UV Laser," Proc. ICCF-8, Lerici, Italy, pp. 287-292, May 21-26 (2000).

11. A.G. Lipson, A.S. Roussetski, C.H. Castano, and G.H. Miley, "In-situ Long - range Alpha Particles and Xray Detection for Thin-film Pd Cathodes During Electrolysis in $\mathrm{Li}_{2} \mathrm{SO}_{4} / \mathrm{H}_{2} \mathrm{O}$," Bult. APS, APS Spring Mtg., Indianapolis, IN, vol. 47, no. 1,Pt. II, p. 1219, Mar 17-22 (2002).

12. A.G. Lipson, A.S. Roussetski, G.H. Miley, and C.H. Castano, "In-situ Charged Particles and X-ray Detection in Pd Thin-film Cathodes During Electrolysis in $\mathrm{Li}_{2} \mathrm{SO}_{4} / \mathrm{H}_{2} \mathrm{O}$," Proc. ICCF-9, Beijing, China, pp. 218-223, May 19-24, (2002).

13. H. Hora, J. C. Kelly, J. U. Patel, M.A. Prelas, G. H. Miley and J. W. Tompkins, "Screening in Cold Fusion Derived from D-D Reactions," Physics Ltrs. A, 175, no. 2, pp.138-143, (1993).

14. G.H. Miley, H. Hora, A. Lipson, S-O Kim, N. Luo, C.H. Castano and G. T. Woo, "Progress in Thin-Film LENR Research," Proc. ICCF-9, Beijing, China, pp. 255-260, May 19-24 (2002). 
15. R. Notoya, "Nuclear Products of Cold Fusion caused by Electrolysis in Alkali Metallic Ions Solutions," Proc. ICCF-5, Monte-Carlo, Monaco, pp.531-538, Apr 9-13 (1995).

16. R. Bush and R, Eagleton, "Evidence for Electrolytically Induced Transmutation and Radioactivity Correlated with Excess Heat in Electrolytic Cells with Light Water Rubidium Salt Electrolytes," Trans. Fusion Tech., vol. 26, pp-344-354, Dec (1994).

17. T.O. Passell, and R.George, "Trace Elements Added to Palladium By Exposure To Gaseous Deuterium," Proc. ICCF-8, Lerici, Italy, pp. 129-134, May 21-26 (2000).

18. H. Yamada, S. Narita, H. Onodera, H. Suzuki, N. Tanaka, T. Nyui and T. Ushirozawa, “Analysis by Timeof-flight Secondary Ion Mass Spectroscopy for Nuclear Products in Hydrogen Penetration through Palladium,"ICCF10-Abstract, Cambridge, MA, p. 136, Aug 25-29 (2003).

19. X. L. Jiang, C. Y. Chen, and D. F. Fu, “Tip Effect And Nuclear-Active Sites," ICCF-7, Vancouver, Canada, pp.175-179, Apr 19-24 (1998).

20. X.Z. Li, Y. Deng, Y.J. Yan, H.F. Huang, W.Z. Yu and C.X. Li, "Nuclear Transmutation Detection in Pd/C Catalyst," J. Energy, vol. 6, no 1, pp.80-86, Summer, (2001).

21. J. Dufour, D. Murat, X. Dufour, and J. Foos, "Hydrex Catalyzed Transmutation of Uranium and Palladium. Experimental Part," Proc. ICCF-8, Lerici, Italy, pp. 153-158, May 21-26 (2000).

22. A. De Ninno, M. V. Antisari and C. Giamgiordano, "Material Science Studies aimed to the Excess Heat Experiments Reproducibility Improvement," ICCF 7, Vancouver, Canada, p. 51, Apr 19-24 (1998).

23. V. Violante, "Analysis of Ni-Hydride Thin Film after Surface Plasmons Generation by Laser Technique," ICCF 10, Cambridge, MA, Aug 25-29 (2003).

24. V. Nassisi and M.L. Longo, "Experimental results of transmutation of elements observed in etched palladium samples by an excimer laser," Fusion Tech., vol.37, No. 3, pp.247-252 (2000).

25. R. Notoya, O. Toshiyuki and N. Yoichi, "Products of Nuclear Processes caused by Electrolysis on Nickel and Platinum Electrodes in Solutions of Alkali-Metallic Ions," ICCF-7, Vancouver, Canada, pp.269-273, Apr 19-24 (1998).

26. A.Takahashi, M. Ohta and T. Mizuno, "Production of Stable Isotopes by Selective Channel Photofission of Pd," J. Applied Physics, vol. 40, pp. 7031-7046, Jap. Soc. Applied Phys., (2001).

27. T. Hanawa, "X-ray Spectrometric Analysis of Carbon Arc products in Water," Proc. ICCF-8, Lerici, Italy, pp. 147-152, May 21-26 (2000).

28. H. Kozima, "CF-Matter and Cold Fusion Phenomenon," Proc. ICCF10, Cambridge, MA, to be published, Aug 24-29, (2003).

29. A.B. Karabut, "Excess Heat Power, Nuclear Products and X-ray Emission in Relation to the High Current Glow Discharge Experimental Parameters," Proc. ICCF9, Beijing, China, May 19-24 (2002).

30. I.B. Savvatimova, A.D. Senchukov and I.P. Chernov, "Transmutation Phenomena in the Palladium Cathode after Ion' irradiation at Glow Discharge," ICCF-6, Hokkaido, Japan, pp.144, Oct 12-13 (1996).

31. I.P. Chernov, N.N. Nikitenkov, L.N. Puchkareva, R. Yu, Kolobov, M. Krening, and H. Baumbach, "Change of Isotopic Composition of Metals at Deuterium Charge," ICCF7, Vancouver, Canada, p.42, Apr 19-24 (1998).

32. J. Dash, I. Savvatimova, S. Frantz, E.Weis, and H. Kozima, "Effects of Glow Discharge with Hydrogen isotope plasmas on radioactivity of Uranium," Proc. ICCF-9, Beijing, China, May 19-24 (2002).

33. G. H. Miley and P. J. Shrestha, "Evidence For Transmutation Reactions in Solids", ANS Mtg, San Diego, CA, Jun (2003).

34. J. Dash, "Review of Experimental Measurements involving Transmutation Reactions," ICCF-10, Cambridge, MA, Aug 25-29, (2003).

35. G.H. Miley, G. Narne, M.J. Williams, J.A. Patterson, J. Nix, D. Cravens and H. Hora, "Quantitative Observation of Transmutation Products Occurring in Thin-Film Coated Microscopheres During Electrolysis," Progress in New hydrogen, pp. 629-644, (1997).

36. A. Lipson, A.S. Roussetski, A.B. Karabut and G.H. Miley, "Strong Enhancement of DD-reaction accompanied by X-ray Generation in a Pulsed Low Voltage High-Current Deuterium Glow Discharge with a Ti-Cathode," ICCF 10, Abstract, Cambridge, MA, p. 90, Aug 24-29, (2003).

37. R. Stringham, "The Cavitation Micro Accelerator," Proc. ICCF-8, Lerici, Italy, pp. 299-304, May 21-26 (2000).

38. T. Ohmori, T. Mizuno, H. Minagawa and M. Enyo, "Low Temperature Nuclear Transmutation Forming Iron On/In Gold Electrode During Light Water Electrolysis," Int. J. Hydrogen Energy, vol. 22, no. 5, pp.459-463, (1997).

39. T. Ohmori and T. Mizuno, "Observation of the Product Elements of Nuclear Transmutation Reaction on/in Several Metal Electrodes by the Cathodic Electrolysis in Light Water Solutions," ICCF7, Vancouver, Canada, p.109, Apr 19-24 (1998). 
40. T. Ohmori and T. Mizuno, "Nuclear Transmutation Reaction Caused By The Light Water Electrolysis On Tungsten Cathode Under An Incandescent Condition,” J. New Energy, vol.4, no. 4, pp.66-78 (2000).

41. T. Ohmori, H. Yamada, S. Narita and T. Mizuno, "Excess Energy and Anomalous Concentration of ${ }^{41} \mathrm{~K}$ Isotopes in Potassium formed on/in a Re Electrode during the Plasma Electrolysis in $\mathrm{K}_{2} \mathrm{CO}_{3} / \mathrm{H}_{2} \mathrm{O}$ and $\mathrm{K}_{2} \mathrm{CO}_{3} / \mathrm{D}_{2} \mathrm{O}$ Solutions," Proc. ICCf-9, Beijing, China, pp. 284-289, May 19-24 (2002).

42. A. Lipson, G. Miley, and A Roussetski, "Energetic Alpha and Proton Emissions on the Electrolysis of Thin-Pd Films" ANS Mtg, San Diego, CA, Jun (2003).

43. R.A. Oriani and J.C. Fisher, "Energetic Charged Particles Detected in the Vapor in Electrolysis Cells," ICCF-10 Abstracts, Cambridge, MA, p. 106, Aug 25-29, (2003).

44. R.A. Oriani and J.C. Fisher, "Detection of Energetic Charged Particles During Electrolysis," ICCF-10 Abstracts, Cambridge, MA, p. 105, Aug 25-29, (2003).

45. G. H. Miley, "On the Reaction Product and Heat Correlation for LENRs", Proc. ICCF-8, Lerici, Italy, pp. 419-424, May 21-26, (2000).

46. J. Dash, G. Noble, and D. Diman, "Surface morphology and microcomposition of Palladium cathodes after electrolysis in acidified light and heavy water: correlation with excess heat," Trans. Fusion Tech., vol. 26, pp. 299-306, Dec, (1994).

47. T. Mizuno, "Nuclear Transmutation: The Reality of Cold Fusion," Infinite Energy Press, (1998).

48. E. Lewis, "Characteristics and Effects of Ball Lightning," ICCF-10 Abstracts, Cambridge, MA, p. 85, Aug 25-29, (2003).

49. K. Shoulders, "Low Voltage Nuclear Transmutation," ICCF-10 Abstracts, Cambridge, MA, p. 117, Aug 25-29, (2003).

50. H. Fox and V. Baraboshkin, "A Review of High-Density Charge Clusters," ICCF9-Abstracts, Beijing, China, p.23, May 19-24, (2002).

51. E. Storms, "What are the conditions required to initiate the LENR Effect?," ICCF-10 Abstracts, Cambridge, MA, p. 121, Aug 25-29, (2003).

52. M. Di Giulio, E. Filipppo, D. Manno, and V. Nassisi, “Analysis of nuclear transmutation observed in Dand H- loaded Pd films," Int'l J. Hydrogen Energy, vol.27, pp.527-531 (2002).

53. H. Hora, J. Kelly, and G. Miley, "Nuclear Shell Magic Numbers Fitting Low-Energy Nuclear Reaction Experiments," Trans. ANS (1998) Annual Mtg, p. 90-92, Nashville, TN, vol. 34, No. 3, Pt 1, Jun 7-11, (1998).

54. M. Wiescher, "Astrophysics of Nuclear Rich Nuclei," $3^{\text {rd }}$ Int'l Conf. Fission \& Properties of Neutron-Rich Nuclei, Sanibel Island, Fl, Nov 3-9 (2002).

55. H. Hora and G. H. Miley, "New Magic Numbers from Low Energy Nuclear Transmutations Predict Element ${ }^{306} \mathrm{X}_{126}$ for Compound Reactions", Czech. J. Physics, vol. 48, No. 9, pp 111-116 (1998).

56. H. Hora, G.H. Miley, J. C. Kelly, G. Selvaggi, A. Tate, F. Osman, R. Castillo, "Proton-Metal Reactions in Thin Films with Boltzmann Distribution Similar to Nuclear Astrophysics”, Fusion Tech., vol. 36, no. 3, pp. 331-336 (1999).

57. H. Hora and G. Miley, "Heavy Nuclide Synthesis by Neutrons in Astrophysics and by screened protons in host metals," Czech. J. Phys., Vol. 50, No. 3, pp 433-439 (2000).

58. G.H. Miley, N. Luo, H. Hora, A. G. Lipson, "Screening Effects in Low Energy Nuclear Reactions of Importance to Astrophysics" IEEE Nuclear Science Symp., paper NS-101, Portland, OR, Oct 19-25, (2003).

59. J. C. Fisher, "Liquid-Drop Model for Extremely Neutron Rich Nuclei," Fusion Tech., vol. 34, no. 1, pp.6675, Aug (1998).

60. J.J. Dufour, J.H. Foos and X.J.C. Dufour, "Formation and Properties of Hydrex and Deutex," Asti Workshop on Anomalies in Hydrogen/Deuterium Loaded Metals, W.J.M.F. Collis, Editor, pp. 29-34, Italy, Nov 27-30 (1997).

61. G. Stoppini, "Nuclear Processes in Hydrogen-Loaded Metals," Fusion Tech., vol. 34, no. 1, pp.81-85, Aug (1998).

62. G. H. Miley and H. Hora, "Nuclear Reactions in Solids," APS 2002 Fall Nuclear Phys. Mtg., East Lansing, MI, Oct 7-10 (2002).

63. G. H. Miley and H. Hora, "Study of Compound Nuclei Produced in Low Energy Nuclear Reactions in Solids," $3^{\text {rd }}$ Intl. Conf. Fission \& Properties of Neutron-Rich Nuclei, Sanibel Island, FL, Nov. 3-9, (2002).

64. N. Luo, C.H. Castano, S-O. Kim, A.G. Lipson, T. Woo, and G.H. Miley, "In-Situ Characterization of Sputtered Thin-Film Electrodes During Electrolysis," Proc. ICCF- 9, Beijing, China, pp. 229-232, May 1924, (2002).

65. N. Luo and G.H. Miley, "First principles studies on the ionic and electronic transport of palladium hydride/deuteride", ICCF 10, Cambridge, MA, Aug 24-29, (2003). 
66. M. McKubre, F. Tanzella, P. Tripodi and P. Hagelstein, "The Emergence of a Coherent Explanation for Anomalies Observed in D/Pd and H/Pd Systems: Evidence for ${ }^{4} \mathrm{He}$ and ${ }^{3} \mathrm{H}$ Production," Proc. ICCF8, Lerici, Italy, pp. 3-10, May 21-26 (2000).

\section{Appendix A}

References employed in compiling Figure 4.

A1. C.G. Beaudette, "Excess Heat Why Cold Fusion Research Prevailed," Oak Grove, Maine, pp.241-246, (2000).

A2. J. O'M Bockris and Z. Minevski, "Two zones of "Impurities" Observed after Prolonged Electrolysis of Deuterium on Palladium," Infinite Energy, no. 5 \& 6, pp. 67-73, Nov (1995).

A3. Castellano, M. Di Giulio, M. Dinescu, V. Nassisi, A. Conte, and P.P. Pompa, "Nuclear Transmutation in Deuterated Pd Films Irradiated by an UV Laser," Proc. ICCF-8, Lerici, Italy, pp. 287-292, May 21-26 (2000).

A4. I.P. Chernov, N.N. Nikitenkov, L.N. Puchkareva, R. Yu, Kolobov, M. Krening, and H. Baumbach, "Change of Isotopic Composition of Metals at Deuterium Charge," ICCF7, Vancouver, Canada, p.42, Apr 19-24 (1998).

A5. J. Dash, G. Noble, and D. Diman, "Surface Morphology and Microcomposition of Palladium Cathodes after Electrolysis in Acidified Light and Heavy Water: Correlation with Excess Heat," Trans. Fusion Tech., vol. 26, pp. 299-306, Dec (1994).

A6. J. Dash, R. Kopecek and S. Miguet, "Excess Heat and Unexpected Elements from Aqueous Electrolysis with Titanium and Palladium Cathodes," Proc. $32^{\text {nd }}$ Intersociety Energy Conversion Engr. Conf., vol. 2, pp. 1350-1355, (1997).

A7. J. Dash, I. Savvatimova, S. Frantz, E. Weis and H. Kozima, "Effects of Glow Discharge with Hydrogen Isotope Plasmas on Radioactivity of Uranium,” Proc. ICCF-9, Beijing, China, May 19-24 (2002).

A8. M. Di Giulio, E. Filipppo, D. Manno and V. Nassisi, "Analysis of Nuclear Transmutation observed in Dand H- loaded Pd films," Int'l J. Hydrogen Energy, vol.27, pp.527-531 (2002).

A9. J.J. Dufour, J.H. Foos and X. J.C. Dufour, "Formation and Properties of Hydrex and Deutex," Asti Workshop on Anomalies in Hydrogen/Deuterium Loaded Metals, pp. 29-34, Italy, (1997).

A10. J. Dufour, D. Murat, X. Dufour and J. Foos, "Hydrex Catalyzed Transmutation of U and Pd. Experimental Part," Proc. ICCF-8, Lerici, Italy, pp. 153-158, May 21-26 (2000).

A11. T. Hanawa, "X-ray Spectrometric Analysis of Carbon Arc products in Water," Proc. ICCF-8, Lerici, Italy, pp. 147-152, May 21-26 (2000).

A12. Iwamura Y., Itoh, T., Gotoh, N., Sakano, M., Toyoda, I., and Sakata, H., "Detection of Anomalous elements, X-ray and Excess Heat induced by Continuous Diffusion of Deuterium through Multi-Layer cathode (Pd/CaO/Pd)," Pro. ICCF-7, Vancouver, Canada, pp. 167-171, Apr 19-24 (1998).

A13. Y. Iwamura, T. Itoh, and M. Sakano, "Nuclear Products And Their Time Dependence Induced By Continuous Diffusion Of Deuterium Through Multi-Layer Palladium Containing Low Work Function Material," Proc. ICCF-8, Lerici, Italy, pp. 141-146, May 21-26 (2000).

A14. Y. Iwamura, T. Itoh, M. Sakano and S. Sakai, "Observation of Low Energy Nuclear Reactions Induced By $\mathrm{D}_{2}$ Gas Permeation Through Pd Complexes," ICCF-9, Beijing, China, May 19-24 (2002).

A15. X.L. Jiang, C.Y. Chen and D. F. Fu, “Tip Effect And Nuclear-Active Sites,” Proc. ICCF-7, Vancouver, Canada, pp.175-179, Apr 19-24 (1998).

A16. A. B. Karabut, "Analysis of Experimental Results on Excess Heat Power production, Impurity Nuclides yield in the Cathode material and penetrating radiation in experiments with high-current Glow Discharge," Proc. ICCF-8, Lerici, Italy, pp. 329-334, May 21-26 (2000).

A17. A. B. Karabut, "Excess Heat Power, Nuclear Products and X-ray Emission in Relation to the High Current Glow Discharge Experimental Parameters," Proc. ICCF9, Beijing, China, May 19-24 (2002).

A18. L.C. Kong, X.L. Han, S.X. Zheng, H.F. Huang, Y.J. Yan, Q.L. Wu, Y. Deng, S.L. Lei, C.X. Li and X.Z. Li, "Nuclear Products And Transmutation In a Gas-Loading D/Pd and H/Pd System," J. Energy, vol.3, no 1, pp.20-23, Spring (1998).

A19. X.Z. Li, Y.J. Yan, T. Jian, M.Y. Mei, Y. Deng, W.Z. Yu, G.Y. Tang, and D. Cao, "Nuclear Transmutation in Pd Deuteride," Proc. ICCF-8, Lerici, Italy, pp. 123-128, May 21-26 (2000).

A20. X.Z. Li, Y. Deng, Y.J. Yan, H.F. Huang, W.Z. Yu and C.X. Li, "Nuclear Transmutation Detection in Pd/C Catalyst," J. Energy, vol. 6, no 1, pp.80-86, Summer (2001).

A21. S. Miguet and J. Dash, "Microanalysis of Palladium after Electrolysis in heavy water," J. New Energy, vol. 1, no. 1, pp. 23-27, (1996). 
A22. G.H. Miley and J.A. Patterson, "Nuclear Transmutation in Thin-film Coatings Undergoing Electrolysis," J. New Energy, vol. 1, pp.11-15, (1996).

A23. G.H. Miley, G. Narne, M.J. Williams, J.A. Patterson, J. Nix, D. Cravens and H. Hora, "Quantitative Observation of Transmutation Products Occurring in Thin-Film Coated Microscopheres During Electrolysis," Progress. in New hydrogen, pp. 629-644, (1997).

A24. G.H. Miley, "Product Characteristics and Energetics in Thin-Film Electrolysis Experiments," Proc. ICCF-7, Vancouver, Canada, pp.241-246, April 19-24, (1998).

A25. T. Mizuno, T. Ohmori, T. Akimoto, K. Kurokawa, M. Kitaichi, K. Inoda, K. Azumi, S. Simokawa and M. Enyo, "Isotopic Distribution For The Elements Evolved In Palladium Cathode After Electrolysis in $\mathrm{D}_{2} \mathrm{O}$ Solution," ICCF-6, Hokkaido, Japan, pp.71, Oct 12-13 (1996).

A26. T. Mizuno, T. Ohmori and M. Enyo, "Anomalous Isotopic Distribution in Palladium Cathode after Electrolysis," Infinite Energy, vol.2, no.7, pp.10-13, (1996).

A27. T. Mizuno, T. Ohmori and M. Enyo, "Isotopic Changes of the Reaction Products Induced by Cathodic Electrolysis in Pd," J. New Energy, vol. 1, no. 3, pp. 31-45, (1996).

A28. T. Mizuno, T. Ohmori and T. Akimoto, T, "Detection of Radiation Emission, Heat generation and Elements from a Pt Electrode induced by Electrolytic Discharge in Alkaline Solutions," Proc. ICCF-7, Vancouver, Canada, pp. 253-258, April 19-24 (1998).

A29. T. Mizuno, "Experimental confirmation of the Nuclear Reaction at Low Energy caused by Electrolysis in the Electrolyte," Proc. Symp. Advanced Research in Energy Tech., Hokkaido University, (2000)

A30. D.W. Mo, Q.S. Cai, L.M. Wang and X.Z. Li, "The Confirmation of Nuclear Transmutation Phenomenon in a Gas Loading H/Pd System Using NAA," ICCF-7, Vancouver, Canada, pp.105, Apr 19-24, (1998).

A31. D.W. Mo, Q.S. Cai, L.M. Wang and S.Z. Wang, "The evidence of nuclear transmutation phenomenon in Pd-H System using NAA (Neutron Activation Analysis)," Proc. ICCF-7, Vancouver, Canada, pp. 259263, Apr 19-24 (1998).

A32. V. Nassisi, "Transmutation of Elements in Saturated palladium Hydrides by an XeCl Excimer Laser," Fusion Tech., vol. 33, no 4, pp.468-475, Jul (1998).

A33. V. Nassisi and M.L. Longo, "Experimental results of transmutation of elements observed in etched Palladium samples by an Excimer Laser," Fusion Tech., vol.37, no. 3, pp.247-252 (2000).

A34. R. Notoya, "Nuclear Products of Cold Fusion caused by Electrolysis in Alkali Metallic Ions Solutions," Proc. ICCF-5, Monte-Carlo, Monaco, pp.531-538, Apr 9-13 (1995).

A35. R. Notoya, O. Toshiyuki and N. Yoichi, "Products of Nuclear Processes caused by Electrolysis on Nickel and Platinum Electrodes in Solutions of Alkali-Metallic Ions," Proc. ICCF-7, Vancouver, Canada, pp.269-273, Apr 19-24 (1998).

A36. T. Ohmori, T. Mizuno and M. Enyo, "Isotopic Distribution of Heavy metal elements produced during the Light Water Electrolysis on Gold Electrode," J. New Energy, vol.1, no. 3, pp. 90-99, (1996).

A37. T. Ohmori, T. Mizuno, H. Minagawa and M. Enyo, "Low Temperature Nuclear Transmutation Forming Iron on/in Gold Electrode during Light Water Electrolysis," Int. J., Hydrogen Energy, vol. 22, no. 5, pp. 459-463, (1997).

A38. T. Ohmori, T. Mizuno, Y. Nodasaka and M. Enyo, "Transmutation In a Gold-Light Water Electrolysis System,” Fusion Tech., vol. 33, no. 3, pp.367-382, May (1998).

A39. T. Ohmori and T. Mizuno, "Observation of the Product Elements of Nuclear Transmutation Reaction on/in Several Metal Electrodes by the Cathodic Electrolysis in Light Water Solutions," ICCF-7, Vancouver, Canada, p.109, Apr 19-24 (1998).

A40. T. Ohmori and T. Mizuno, "Nuclear Transmutation Reaction Caused By The Light Water Electrolysis On Tungsten Cathode Under An Incandescent Condition," J. New Energy, vol.4, no. 4, pp.66-78 (2000).

A41. T. Ohmori and T. Mizuno, "Strong Excess Energy Evolution, New Elements Production, and Electromagnetic Wave and/or Neutron Emission in the Light Water Electrolysis with a Tungsten Cathode," Proc. ICCF-7, Vancouver, Canada, pp. 279-284, Apr 19-24 (1998).

A42. T. Ohmori, H. Yamada, S. Narita and T. Mizuno, "Excess Energy and Anomalous Concentration of ${ }^{41} \mathrm{~K}$ Isotopes in Potassium formed on/in a Re Electrode during the Plasma Electrolysis in $\mathrm{K}_{2} \mathrm{CO}_{3} / \mathrm{H}_{2} \mathrm{O}$ and $\mathrm{K}_{2} \mathrm{CO}_{3} / \mathrm{D}_{2} \mathrm{O}$ Solutions," Proc. ICCf-9, pp. 284-289, May 19-24 (2002).

A43. T.O. Passell and R. George, "Trace Elements Added to Palladium By Exposure To Gaseous Deuterium," Proc. ICCF-8, Lerici, Italy, pp. 129-134, May 21-26 (2000).

A44. I.B. Savvatimova, Y.R. Kuchernov and A.B. Karabut, "Cathode Material change after Deuterium Glow Discharge Experiments," Trans. Fusion Tech., vol. 26, p. 389-394, Dec (1994).

A45. I.B. Savvatimova, A.D. Senchukov and I.P. Chernov, "Transmutation Phenomena in the Palladium Cathode after Ion irradiation at Glow Discharge," ICCF-6 Hokkaido, Japan, pp.144, Oct 12-13 (1996). 
A46. J. Warner and J. Dash, J., "Electrolysis of $\mathrm{D}_{2} \mathrm{O}$ with Titanium cathodes: Enhancement of excess heat and further evidence of possible transmutation," ICCF-9, Beijing, China, pp. 118-119, May 19-24 (2002).

A47. H. Yamada, K. Uchiyama, N. Kawata, Y. Kurisawa and M. Nakamura, "Producing a radioactive source in a deuterated Palladium electrode under direct-current Glow Discharge," Fusion Tech., vol. 39, pp. 253258, Mar (2001).

A48. H. Yamada, S. Narita, Y. Fujii, T. Sato, S. Sasaki and T. Ohmori, "Production of Ba and several Anomalous Elements in Pd under light Water Electrolysis," ICCF-9, Beijing, China, p. 123, May 19-24 (2002). 